Limit Theorems for Random Fields with Singular Spectrum 


\section{Mathematics and Its Applications}

Managing Editor:

M. HAZEWINKEL

Centre for Mathematics and Computer Science, Amsterdam, The Netherlands 


\section{Limit Theorems}

for Random Fields

with Singular Spectrum

by

Nikolai Leonenko

Department of Mathematics,

University of Kiev,

Kiev, Ukraine

SPRINGER-SCIENCE+BUSINESS MEDIA, B.V. 
A C.I.P. Catalogue record for this book is available from the Library of Congress.

ISBN 978-94-010-5947-3 ISBN 978-94-011-4607-4 (eBook)

DOI 10.1007/978-94-011-4607-4

Printed on acid-free paper

All Rights Reserved

C1999 Springer Science+Business Media Dordrecht

Originally published by Kluwer Academic Publishers in 1999

No part of the material protected by this copyright notice may be reproduced or utilized in any form or by any means, electronic or mechanical, including photocopying, recording or by any information storage and retrieval system, without written permission from the copyright owner 


\section{Contents}

1 SECOND-ORDER ANALYSIS OF RANDOM FIELDS 1

1.1 Basic Concepts and Notation . . . . . . . . . . . . 1

1.2 Elements of Spectral Theory of Random Fields . . . . . 11

1.3 Models of Random Processes and Fields with Singular Spectrum . . . . . . . . . . . . . . . 38

1.4 Tauberian and Abelian Theorems for Correlation Function of Homogeneous Isotropic Random Fields . . . . . 61

2 LIMIT THEOREMS FOR NON-LINEAR TRANSFORMATIONS OF RANDOM FIELDS

2.1 Some Properties of Gaussian and $\chi$-Squared Random Fields . . . . . . . . . . . . . . . . . 105

2.2 Reduction Theorems for the Local Functionals of Random Fields with Slowly Decaying Correlations . . . . . 112

2.3 Multiple Stochastic Integrals . . . . . . . . . . . . . . . 123

2.4 Non-Central Limit Theorems for Local Functionals of Random Fields . . . . . . . . . . . . . . . . . . . . . . . . . 129

3 ASYMPTOTIC DISTRIBUTIONS OF GEOMETRIC FUNCTIONALS OF RANDOM FIELDS

3.1 Limit Distributions for Characteristics of the Excess above a Level for Gaussian Fields . . . . . . . . . . . 137

3.2 Limiting Distributions for the Excess Over a Radial Surface of $\chi$-Squared Random Fields . . . . . . . . 150

3.3 Spherical Measures of Excess over of Moving Level . . 164

3.4 Sojourns of Multi-Dimensional Gaussian Fields with Dependent Components . . . . . . . . . . . . . . 170 
3.5 Asymptotic Normality of Random 'Area of Surface' of Planar Gaussian Field . . . . . . . . . . . . . . . . 189

3.6 Asymptotics for Occupation Densities of Gaussian and z-Squared Random Fields . . . . . . . . . . . . . . 194

4 LIMIT THEOREMS FOR SOLUTIONS OF THE BURGERS' EQUATION WITH RANDOM DATA

4.1 Physical Motivation and Recent History . . . . . . 211

4.2 Hopf-Cole Solution . . . . . . . . . . . . . . . . . . 213

4.3 Parabolic Asymptotics for Weakly Dependent Random Data : the Gaussian Scenario . . . . . . . . . . 222

4.4 Parabolic Limits for Strongly Dependent Random Initial Conditions: the Gaussian Scenario . . . . . . . . . 243

4.5 Parabolic Limits for Strongly Dependent Random Data : the Non-Gaussian Scenario 253

4.6 Exact Parabolic Asymptotics for Singular Burgers' Equation . . . . . . . . . . . . . . . . 261

4.7 Hyperbolic Asymptotics for Rescaled Solutions of Burgers' Equation . . . . . . . . . . . . . . . 266

5 STATISTICAL PROBLEMS FOR RANDOM FIELDS WITH SINGULAR SPECTRUM

5.1 Estimation of Mathematical Expectation . . . . . . . 275

5.2 Estimation of the Covariance Function . . . . . . . . 306

5.3 Efficient Estimation of Regression Coefficients of a Random Fields Observed on the Sphere . . . . . . . . 335

5.4 Estimation in the Frequency Domain . . . . . . . . . 340

$\begin{array}{lr}\text { Comments } & 350\end{array}$

$\begin{array}{ll}\text { Bibliography } & 357\end{array}$

$\begin{array}{ll}\text { Index } & 394\end{array}$ 


\section{Preface}

This book is devoted to an investigation of the basic problems of the theory of random fields which are characterized by certain singular properties (e. g., unboundedness, or vanishing) of their spectral densities. These random fields are called, the random fields with singular spectrum, long-memory fields, random fields with long-range dependence, fields with slowly decaying correlations or strongly dependent random fields by various authors. This phenomenon has been observed empirically by many scientists long before suitable mathematical models were known. The methods and results differ significantly from the theory of weakly dependent random fields.

The first chapter presents basic concepts of the spectral theory of random fields, some examples of random processes and fields with singular spectrum, Tauberian and Abelian theorems for the covariance function of singular random fields.

In the second chapter limit theorems for non-linear functionals of random fields with singular spectrum are proved.

Chapter 3 summarizes some limit theorems for geometric functionals of random fields with long-range dependence.

Limit distributions of the solutions of Burgers equation with random data via parabolic and hyperbolic rescaling are presented in chapter 4 .

And chapter 5 presents some problems of statistical analysis of random fields with singular spectrum.

I would like to thank the editor, Michiel Hazewinkel, for his support.

I am grateful to the following students and colleagues: I. Deriev, A. Olenko, K. Rybasov, L. Sakhno, M. Sharapov, A. Sikorskii, M. Silac-Benšic.

I would also like to thank V.Anh, O. Barndorff-Nielsen,Yu. Belyaev, P. Biler, R. Bhattacharya, C. Boldrighini, R. Bradley, V. Buldigin, A. Bulinsky, Yu. Daletski,Yu. Davydov, E. Donskoi, M. Farge, R. Gill, L. Giraitis, M. Goria, D. Gurarie, A. Ivanov, M. Kelbert, Yu. Kondratiev, P. Kotelenez, Yu. Kozachenko, Li Zhanbing, M. Maejima, Yu. Mishura, A. Novikov, G. Peskir, V. Petrov, M. Priestley, E. Orsingher, N. Portenko, Yu. Prokhorov, Yu. Samoilenko, K. Schneider, O. Seleznev, A. Shiryaev, Yu. Suhov,V. Statulevičius, D. Surgailis, M. Taqqu, G. Terdik, E. Valkeila, A. Veretennikov, W. Woyczynski for many interesting discussions on random fields.

I consider it my pleasent duty to express here my deep gratitude to A. Skorokhod.

I learned theory of random fields from M.Yadrenko. I thank him now for all the help and encouragement he has provided. 
I would like to thank my son Eugene and daughter Anna for their support. Finally, many thanks to my wife Olga for her understanding and help.

N. Leonenko

Kiev, Ukraine

April 1998. 